\title{
The stress test: Can stress ever be beneficial?
}

\author{
Joint British Academy-British Psychological Society Lecture \\ read 22 September 2016
}

\section{IAN H ROBERTSON}

Trinity College Dublin

Abstract: The human brain needs an optimal amount of challenge in order to perform optimally. This is because challenge activates the neurotransmitter noradrenaline (NA) which, at optimal levels, facilitates good brain performance; in contrast, when levels of NA are either too low (sleepy, jaded, bored, mood low) or too high (stressed, anxiety, pressure), the brain underperforms because of the key neuromodulatory effect of noradrenaline. Moderate amounts of stress can actually increase performance and attention to such a 'sweet spot' and hence to higher levels of cognitive performance with resulting improved confidence. Stress arises when people perceive the demands made upon them to exceed their ability to cope with these demands. But this in turn depends upon appraisal of the stress and in particular whether the stressors are seen as challenges to be overcome or threats to be avoided. A 'challenge' mindset makes it easier for individuals to use adversity to attain this optimal zone of performance and build resilience. Furthermore, moderately stressful events in a person's life have a number of potentially beneficial effects including emotional resilience, better chronic pain tolerance and even enhanced cognitive function.

Keywords: stress, anxiety, mood, attention, adversity, resilience, cognitive function, chronic pain.

Attitudes to stress have changed considerably over the last hundred years in the developed world. Anecdotal accounts of parenting and schooling from the first part of the 20th century are replete with tales of harsh treatments that aimed to 'toughen' children and adolescents, with corresponding attitudes to adults. The inevitable psychological casualties of such regimes, along with the growth of psychological and sociological research, eventually helped trigger a more person-centred approach to psychological vulnerabilities that came to prevail, in the Western world at least, during the late 20 th and early 21 st centuries. 
Remarkably, however, this change has coincided with a very significant increase in diagnosed psychological problems linked to perceived stress. Stress can be defined as the perception that the demands made upon one exceed one's resources to cope with these. The resulting emotion is anxiety. Almost one in five UK citizens are estimated to suffer a significant anxiety disorder in any one year (Fineberg et al. 2013), with a similar estimated prevalence in the USA (Kessler et al. 2005). Chronic anxiety lowers mood and there has been a remarkable escalation in the prescription of SSRI (selective serotonin re-uptake inhibitors) drugs across the developed world for the treatment of both anxiety and mood disorders.

For example, the population of England in 2013 was 53 million and during that year the number of prescriptions for antidepressants was 53 million (most of which would be repeat prescriptions and not just new ones). Just two years later, in 2015, this figure had risen to 61 million (http://content.digital.nhs.uk), which represents a doubling in a decade.

This remarkable level of mass-medication of populations represents a considerable challenge in understanding stress - increasing treatment should surely reduce the disorder, whereas in contrast we are seeing a progressive rise. According to the World Health Organization, depression is now the single leading cause of disability worldwide (http://www.who.int/mediacentre/factsheets/fs369/en/).

How are these two social phenomena - the abandonment of the 'grin and bear it' approach to psychological distress on the one hand, and the massive expansion of diagnosis and treatment on the other-related, if at all?

One possibility is that the actual level of psychological distress has not increased, but rather it is being diagnosed more readily. This argument does not hold up, however, because self-reported mood has actually been declining over the last 70 years in the USA, at least. The average score on a standard measure of depressed mood (MMPI depression scale) among students has risen steadily over that period-by around 20 per cent on average (Twenge et al. 2010).

Young Westerners, then, report themselves to be significantly less happy now than they did 70 years ago, and hence under more stress. There are a number of sociocultural-economic reasons for this which are neither fully understood nor within the scope of the present paper to explore.

In this paper, I set out the evidence that one key psychological factor may play a part in this epidemic of distress - namely a failure to understand and embrace the positive effects that moderate levels of stress can have on mind, body and emotions.

This seemingly counter-intuitive approach to moderate stress understandably runs against the grain of a mental health movement that seeks to minimise stress and anxiety, if for no other reasons than of awakening unpleasant resonances of the 'grin and bear it' approach to psychological distress that pertained in the first half of the 20th century. 
Yet the evidence, as will be seen in the course of this paper, is quite strong that properly embraced, moderate adversity and its associated stress can have quite significant beneficial effects which, if appreciated and used, could help individuals harness moderate stress rather than seek to avoid it and have it medicalised.

A related question is whether the medicalisation of stress represented by antidepressant prescribing figures causes as many problems as it solves? Is stress always something to be treated - or can it sometimes have beneficial effects? This paper will examine the extent to which attitudes to, and tolerance of, adversity, can influence psychological well-being. I will also ask whether, under certain circumstances, stress can have positive effects that a possible over-reliance on prescription medications may mask. Answering these questions, however, requires me to address some questions about the human brain and behaviour that ostensibly have little to do with stress.

\section{WHY DO CIVIL ENGINEERS BUILD BENDS IN ROADS WHERE THERE IS NO GEOLOGICAL NEED TO DO SO?}

Civil engineers avoid building long, straight roads because experience has taught them that unless curves are built into them, drivers tend to lose concentration and drive off them (The Highways Agency 2016). This is because sustained attention is one of the hardest tasks for the human brain to achieve and is the major cause of accidents not just on roads, but also on railways across the world when train drivers travel through red lights due to lapsed attention (Edkins \& Pollock 1997).

Attention refers to the capacity to control, moment to moment, the contents of awareness. There are three broad categories of attention (Robertson et al. 1996), the most common being selective attention, which refers to the ability to choose to attend to one strand of information from the external (e.g. one person's voice against a background of many) or internal (e.g. memory of a particular conversation) environment. Selective attention can be deployed across sensory modalities: for example, allowing you to focus your mind on the clarinet against the complex sounds of an orchestra.

The second category of attention is attentional switching, where focus is shifted from one stream of information (e.g. the melody of the clarinet) to another (e.g. the words sung by the accompanying vocal soloist).

Sustained attention is the third category of attention and refers to the ability to keep one's mind on routine tasks and over-ride the brain's tendency preferentially to process novelty and change over predictable repetition.

Sustained attention is hence required in situations that have no intrinsic attentiongrabbing properties such as change, novelty, emotional tone, threat or challenge. Video-games, for example, require little sustained attention because their designers 
are expert at externally controlling our attention through these variables. In contrast, reading a book or listening to a lecture demand that we can keep our mind on the task in hand by internally- rather than externally-driven processes.

My colleagues and I in Dublin and Cambridge found that we could measure sustained attention using a simple computerised test called the SART (Sustained Attention to Response Task) which simply required people to press a button to every number between 1 and 9 which appeared at a rate of just slower than one per second, except the number 3 (Robertson et al. 1997). This apparently very easy task resulted in a surprising number of errors with people uttering 'Oops', or worse, when they found themselves 'tricked' into pressing the 3 when they had not intended to.

This SART turned out to be an excellent laboratory measure of the type of sustained attention needed to keep your mind on a dull, repetitive situation like driving a train on a long straight track. It is also, it turns out, a pretty good measure of people's propensity to make absent-minded slips in everyday life, as colleagues in Canada showed after reviewing the SART literature (Smilek et al. 2010).

We therefore considered the SART to create the laboratory equivalent of the long straight road that makes it so difficult for drivers to concentrate. What is the equivalent of the road with bends it, however? Tom Manly and I tested the hypothesis that if you increased the proportion of no-go targets in the test, it would be experienced as more difficult, compared to the very low frequency version, because it would involve more frequent switches between press and no-press modes. This is in fact what happenedparadoxically as the test became more 'difficult' (i.e. more like the road with bends in it), the proportion of absent-minded false presses decreased (Manly et al. 1999).

What is more, while the low-frequency (straight-road) SART distinguished clearly between people who were more or less prone to everyday absent-mindedness, the high-frequency (bendy road) task did not, as Figure 1 shows.

Attention Deficit Hyperactivity Disorder (ADHD) is a common childhood complaint that persists in altered form into adulthood in approximately 50 per cent of cases (Bellgrove et al. 2005). A key symptom of this problem is a difficulty in keeping attention focussed on the task in hand, resulting in great distractibility and behavioural difficulties in children, not least in the school classroom. Our research suggests that the sustained attention system is particularly impaired in ADHD, while the selective attention system is relatively spared (Manly et al. 2001).

Noradrenaline (NA) is one of the brain's key neurotransmitters or 'chemical messengers'. It is produced deep in the mid-brain in a tiny structure called the locus coeruleus, and is triggered whenever we are engaged, excited, frightened, alerted, curious, challenged or generally aroused (Sara 2009). NA is a 'neuromodulator', because in the right quantities, it helps different parts of the brain synchronise sweetly with each other to produce fluent performance in mental or physical tasks. 


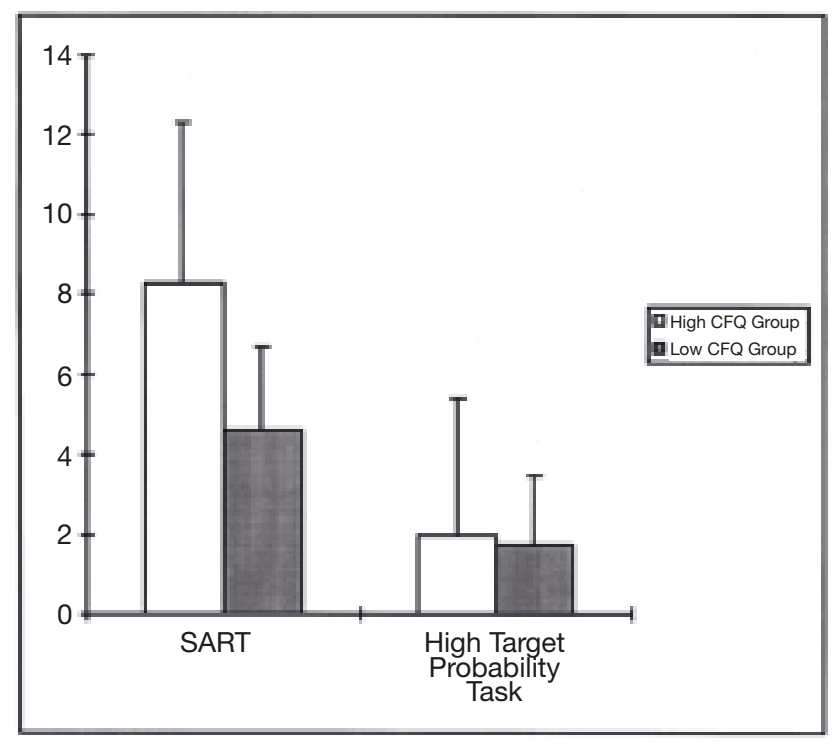

Figure 1. Fewer absent-minded errors as a task becomes more challenging (Manly et al. 1999).

We discovered that both in children with Attention Deficit Hyperactivity Disorder, and in healthy young adults, a highly specific link existed between the availability of noradrenaline in the brain and the ability to sustain attention-in particular the propensity to make SART errors (Bellgrove et al. 2006, Greene et al. 2009).

The prefrontal cortex of the brain, which occupies that region above the eyes and from the ears forward, has long been linked to high-level 'executive' control over our behaviour, where planning, flexibility, judgement and inhibition of irrelevant activity are emphasised. Attention is a crucial part of such complex behaviour, and sustained attention is particulary important in that it involves over-riding more primitive impulses to respond to immediately attractive stimuli in favour of colourless longer-term goals (such as not crashing a train, for example).

We also found that a particular region of the brain - the right prefrontal cortex, was implicated in the ability to sustain attention. We showed this in two separate functional Magnetic Resonance Imaging (fMRI) studies in Cambridge and Toronto where we compared which brain regions were active - in each it turned out to be the right dorsolateral prefrontal cortex - when people were trying to keep their mind on the SART test, compared to when they were not (O'Connor et al. 2011, Manly et al. 2003).

Then, using a quite different methodology, colleagues in Belgium gave the SART to people who had the misfortune to suffer a stroke, and examined damage to which part of the brain best predicted having difficulty doing the SART accurately. These researchers confirmed the fMRI findings by showing a very specific linkage of SART 
errors to right prefrontal cortex lesions (Molenberghs et al. 2009). A subsequent meta-analysis of scores of different studies of sustained attention tasks confirmed the involvement of the right dorsolateral prefrontal cortex in sustaining attention to routine, boring tasks (Singh-Curry \& Husain 2009).

But had these two disparate correlates of the SART - right prefrontal cortex on the one hand, and the neurotransmitter noradrenaline on the other-anything to do with each other? A review of the literature showed that indeed they had-studies which used drugs to increase activity specifically in the noradrenaline system consistently found that the brain region that increased its activity in response was the right dorsolateral prefrontal cortex (Bush et al. 2012, Grefkes et al. 2010, Chamberlain et al. 2007).

One major challenge of following through on this link between sustained attention and noradrenaline is that it has not been possible directly to observe NA activity in the waking human brain. We were able to overcome this hurdle in Dublin where Peter Murphy, Redmond O'Connell, Josh Balsters and I showed that the eye's pupil was an accurate indicator of NA activity in the human brain.

We are familiar with people's pupils dilating in pleasure or attraction, which reflects the brain's increasing NA activity as part of an emotional response. But the pupil also dilates whenever we pay attention to something through being curious, surprised, engaged or challenged. This is quite different from the pupil response to changes in light - for example, the pupil dilating when it becomes dark. This latter pupil response is controlled by the neurotransmitter acetylcholine, while the pupil's 'attention response' is controlled by noradrenaline.

We showed that this was the case by looking at the pupil's response to targets that participants had been trained to pay attention to, while simultaneously measuring the brain's activity using fMRI, in the part of the brain known to produce NA, the locus coeruleus. For the first time, our group demonstrated that whenever the pupil got bigger in response to a target, the locus coeruleus increased its activity.

But one other finding confirmed an early finding in psychology, the YerkesDodson curve (Yerkes \& Dodson 1908). This inverted-U-shaped curve demonstrates an important principle of many human functions, including those of many neurotransmitters, namely that peak performance (say in accuracy of response, or speed of thinking, or reliability of memory) is achieved where there are intermediate levels of 'arousal'. For example, if alertness levels are low because it is very early in the morning, a person may be sluggish in their ability to do mental arithmetic. Similarly, if alertness levels are too high because of high anxiety, arithmetic performance may be similarly impaired. In the median zone, at the peak of the inverted U, there is a 'sweet spot' of performance. 
Animal research has shown that NA and some other neurotransmitters - dopamine, for example - share this inverted-U-shaped function, and this fact in relation to NA was thought to underpin the Yerkes-Dodson curve.

We were able to show for the first time that the human pupil response showed this Yerkes-Dodson-type curve (Murphy et al. 2014). Just before every target, the pupil diameter was measured, and then the quality of attention (measured by the variability of the speed of response, a very sensitive measure of attentional focus) for responses at different pupil diameters measured. We found that small and large pre-target pupil diameters were associated with poor attention, in contrast to medium diameters which were associated with very good sustained attention (Murphy et al. 2011), as seen in Figure 2, where lower scores represent better attentional focus.

So, in addressing the question as to why civil engineers build unnecessary bends in long roads, the answer is that it is to engineer a level of arousal closer to the 'sweet spot' represented by moderate levels of noradrenergic activity in the locus coeruleus. Like many of the brain's neurotransmitters, this inverted-U-shape function dictates that both too low (under-stimulation, low challenge) and high (over-stimulation, very high challenge/stress) levels impair attention.

Driving down an unremittingly straight road places too great a demand on the brain's capacity for sustaining attention and loss of focus and alertness result, with potentially dangerous outcomes. Civil engineers have learned by trial and error that

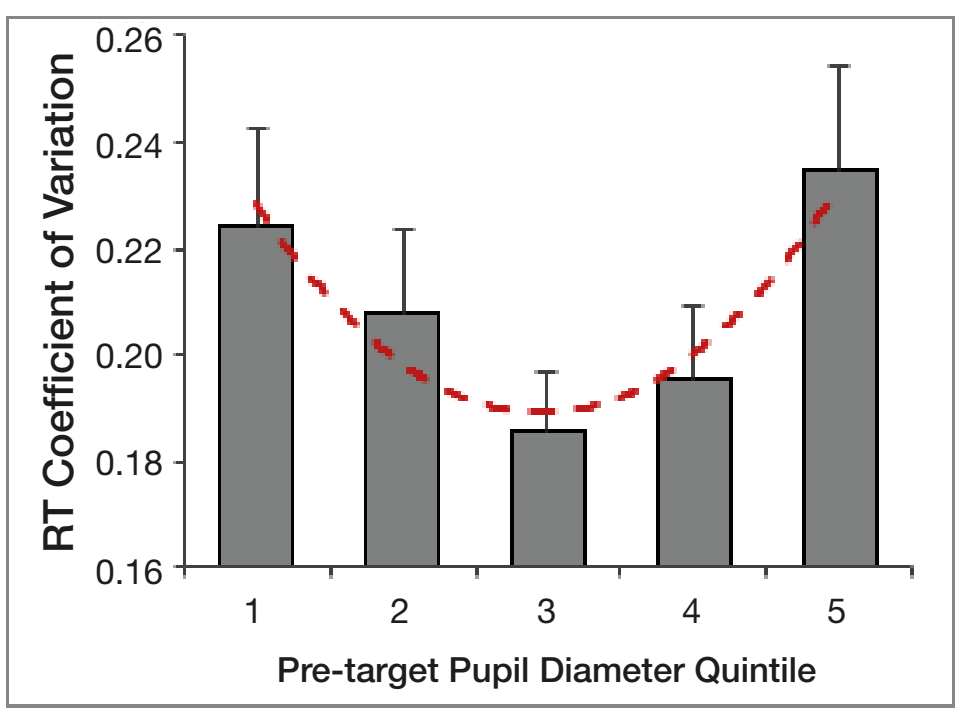

Figure 2. Optimal focus with moderate levels of pupil dilation/noradrenergic function. 
they have to build 'challenge' into their roads in order to trigger the driver's brain to produce sufficient NA to maintain reasonable driving performance.

We were able to put this principle into practice with people suffering from traumatic brain injury (TBI) in whom disruption of frontal lobe activity commonly leads to a number of difficulties, including difficulty in sustaining attention (Robertson et al. 1997). We were able temporarily to improve their cognitive and emotional functioning. We did this using a simulated hotel receptionist job situation where they had to perform a number of different tasks and remember not to neglect any one task through giving equal time to each. On average TBI individuals missed out one complete task - a really poor job performance - compared to control, non-impaired individuals.

We then tried to increase their alertness levels by ringing a buzzer randomly and occasionally during the task - the equivalent of putting a bend in the long straight road. This simple intervention led to them-temporarily, of course - showing normal performance on the receptionist task, probably because their brain was temporarily nearer its optimal level of NA activity due to the arousing stimulus (Manly et al. 2002).

We put this principle into more long-term use in adults with ADHD, many of whom, we had discovered, were chronically short of NA in their brains and hence had major problems with attention. With Redmond O'Connell in Dublin we showed that you could train them to give temporary boosts to their arousal by feeding back to them using a device called a Skin Conductance Response (SCR) device, which measures the skin's sweatiness. This measure is a very good marker of the brain's state of arousal and by allowing people to see what their arousal levels are on the screen of a computer, they can learn to control it up or down, which is the principle of 'biofeedback'.

We trained these adults with ADHD to generate spikes of arousal at important times when they would otherwise be liable to make an absent-minded or impulsive slip (O'Connell et al. 2008) and subsequently we developed a self-administered training programme that produced extended improvement in ADHD symptoms, cognitive function and depression in blind follow-up three months later (Salomone et al. 2015).

These findings led to a second, related, question regarding the relationship between stress and arousal.

\section{WHY IS HIGHER CORTISOL ASSOCIATED WITH BETTER PERFORMANCE IN LOW-MATHS-ANXIETY STUDENTS AND WITH WORSE PERFORMANCE IN HIGH-MATHS-ANXIETY STUDENTS?}

Cortisol is the human stress hormone, part of the body's 'fight or flight' response, and a useful short-term energiser of human performance in conditions where urgent action is required. Short-term, cortisol boosts mental and physical performance, 
but when it is elevated over prolonged periods, it can cause damage to the body and brain.

In any stressful situation - for example, performing in front of a critical audience -it is normal to see cortisol levels go up in healthy people. And this is true in one study where people had to perform mental arithmetic in front of a critical audience-a stressful situation that caused all participants to generate big increases in cortisol (Mattarella-Micke et al. 2011).

All participants were equally arithmetically capable, but half were, even before the experiment, anxious about their ability, while the other half were not.

As might be expected, among the maths-anxious students, the more their cortisol levels surged, the worse they performed. But what was very strange was that the reverse was true for the maths non-anxious students - the more their cortisol levels rose, the better they performed. This is likely because the anxious students were already high in arousal because of this, and the extra arousal created by performing difficult problems in front of an audience pushed them over beyond their 'sweet spot' of functioning, and hence to poorer performance.

The low-maths-anxiety students on the other hand, were probably at lower levels of arousal and the challenge of performance pushed them up towards their 'sweet spot'. Stress for them, therefore, fuelled better performance because of this.

In a much darker domain, stress appeared to bolster cognitive performance in a much more enduring, counter-intuitive way. My colleagues in Dublin working on the Irish Longitudinal Study on Ageing (TILDA) found that roughly one in fifteen of the fifty- to ninety-year-olds reported that they had been sexually abused as children. This wasn't the surprise - such figures are fairly typical of research studies worldwide. As is usually the case, in adulthood these abuse victims were more depressed and also less physically healthy than their contemporaries. This fairly typical finding is explained by the prolonged stress-linked damage to the body caused by being subjected to early abuse. So the picture here fitted with what we already knew, except in one respect: despite their other problems, they were cognitively better on a number of cognitive tests including their ability to remember lists of words, their speed of thinking, and their ability to control their attention ('executive function') than non-abused people in the study of the same age, social and educational background (Feeney et al. 2013) Because of the size of the study - nearly 7,000 people - we could rule out other physical, social and mental factors that might have caused the differences.

Stress also has other unexpected effects amidst human suffering. One Dutch study measured cognitive abilities in more than a thousand people in their early seventies, and then again three years later (Comijs et al. 2011). The seventies is a difficult decade for many, with new pressures on people, such as a spouse succumbing to serious illness. Conflicts with families can also spring up during the major changes that are so 
common at this time of life. The Amsterdam group were no exception-over three quarters of them reported having experienced at least one very significant 'life event' during the three years. And those whose partners had suffered a serious illness showed better cognitive function than those who had not. Furthermore, people who had run into severe conflict with other people - family, friends, neighbours, etc — were also cognitively sharper three years later.

This is very likely because stress raises noradrenaline levels in older people, some of whose relatively undemanding lifestyles might have resulted in low levels of noradrenergic stimulation. The challenge and stress of looking after a seriously ill partner, or the demands of coping with family conflict, may have raised these levels to nearer the optimum 'sweet spot' that is beneficial to cognitive functioning.

These and other findings led me to propose a theory to explain how 'cognitive reserve' functions to reduce risk of dementia. Cognitive reserve is the term coined for a combination of education, intelligence level and mental stimulation that together strongly predict vulnerability to Alzheimer's Disease (Stern et al. 1996). Education level is one of the strongest predictors of reduced risk of dementia, yet there has been no adequate theory of how a social variable like education might influence a biological variable such as Alzheimer's Disease. My noradrenergic theory of cognitive reserve was the first attempt at such a theory.

All forms of mental challenge, including moderate stress, increase noradrenaline (NA) levels in the brain. Providing NA stays near its 'sweet spot', then it not only improves cognitive functioning but also helps the brain form new connections and new brain cells (Robertson 2013), a view that has subsequently been supported in studies in other laboratories (Wilson et al. 2013, Clewett et al. 2016).

But it is not only in old age that stress can be beneficial. Young adults who have experienced moderate levels of adversity in their lives have lower levels of emotional vulnerability than is the case for those who have had no or little adversity (Seery et al. 2010a). Those who have experienced moderate stressors in their life are also much less likely to be disabled and off work if they suffer chronic back pain, compared to those who have suffered little or no adversity (Seery et al. 2010b).

Could this help explain the rising levels of distress across generations? Have more recent generations been protected against adversity in the relatively prosperous and stable Western world that has existed since World War 2? Has the switch to a more 'psychological' approach to stress, contrasting with the 'grin and bear it' attitudes of previous generations, led to a relative over-protection of younger people? If we accept the results by Seery and colleagues about the role of moderate adversity in emotionally toughening younger people against various adult emotional and physical ailments, then this must be seen as a tenable hypothesis. 
Stress, in moderate levels, can have positive emotional, cognitive and physical consequences, but this depends on appropriate cognitive appraisal and a resulting optimal level of arousal. Some adversity appears to be essential in life so that individuals can learn habits of managing stress, but also so that they can experience the optimisation of noradrenaline levels in the brain that properly appraised 'challenge' can cause.

The inverted-U-shaped function of stress - too much or too little can be bad for you - is related to the inverted-U-shaped function of key brain neurotransmitters such as noradrenaline. Given that NA is a key component of the 'fight or flight' stress response, this is not particularly surprising.

The question arises whether in our anxiety as a culture to avoid the harsh 'grin and bear it' attitudes of our forebears, we have lurched too far into a realm where any stress is seen as bad and to be avoided. Has this resulted in Western cultures in over-protection of youngsters from what otherwise might be psychologically strengthening experiences of mild adversity, albeit with some inevitable attendant greater risk?

Linked to this approach to distress are the psychological implications of administering medication to millions of people for their psychological distress. What message does this convey to them about the causes of their emotional difficulties? Does it imply that their emotions are caused by biological factors outside of their control? And if so, does that then sabotage their abilities to benefit from moderate levels of stress and the psychological and physiological learnings-'inoculation'that can arise from them?

Stress can be beneficial to both emotional and cognitive functioning. By realising this fact, and understanding how it happens, we can hope that people may learn to cope better and benefit from the lessons that moderately severe stressors can teach us.

This summary cannot go into the full detail of the fascinating science surrounding the positives of stress and the reader is referred to my book on the subject (Robertson 2016) for a fuller account.

\section{Acknowledgments}

I would like to thank my wonderful colleagues in Cambridge, London, Dublin and Toronto with whom I have worked over the last 35 years. I would also like to thank the British Psychological Society for inviting me to give the annual Joint British Academy - British Psychological Society Lecture which the current paper documents. My grateful thanks to the British Academy for their support, expertise and hospitality. 


\section{REFERENCES}

Bellgrove, M. A., Gill, M., Hawi, Z., Kirley, A. \& Robertson, I. H. (2005), 'Dissecting the Attention Deficit Hyperactivity Disorder (ADHD) Phenotype: Sustained Attention and Spatial Attention Asymmetries in Relation to Dopamine Transporter (DAT1) Transporter', Neuropsychologia, 43: 1847-57.https://doi.org/10.1016/j.neuropsychologia.2005.03.011

Bellgrove, M. A., Hawi, Z., Gill, M. \& Robertson, I. H. (2006), 'The Cognitive Genetics of Attention Deficit Hyperactivity Disorder (ADHD): Sustained attention as a Candidate Phenotype', Cortex, 42: 838-45. https://doi.org/10.1016/S0010-9452(08)70426-X

Bush, G., Holmes, J., Shin, L. M., Surman, C., Makris, N., Mick, E., Seidman, L. J. \& Biederman, J. (2012), 'Atomoxetine Increases Fronto-Parietal Functional MRI Activation in Attention-Deficit/ Hyperactivity Disorder: A Pilot Study', Psychiatry Research, 211: 88-91. https://doi.org/10.1016/j.pscychresns.2012.09.004

Chamberlain, S. R., Campo, N. D., Dowson, J., Müller, U., Clark, L., Robbins, T. W. \& Sahakian, B. J. (2007), Atomoxetine Improved Response Inhibition in Adults With Attention Deficit/ Hyperactivity Disorder', Biological Psychiatry, 62: 977-84.

https://doi.org/10.1016/j.biopsych.2007.03.003

Clewett, D. V., Lee, T.-H., Greening, S., Ponzio, A., Margalit, E. \& Mather, M. (2016), 'Neuromelanin Marks The Spot: Identifying a Locus Coeruleus Biomarker of Cognitive Reserve in Healthy Aging', Neurobiology of Aging, 37: 117-26. https://doi.org/10.1016/j.neurobiolaging.2015.09.019

Comijs, H. C., van den Kommer, T. N., Minnaar, R. W. M., Penninx, B. W. J. H. \& Deeg, D. J. H. (2011), 'Accumulated and Differential Effects of Life Events on Cognitive Decline in Older Persons: Depending on Depression, Baseline Cognition, or ApoE $\varepsilon 4$ Status', The Journals of Gerontology Series B: Psychological Sciences and Social Sciences, 66B: 111-20. https://doi.org/10.1093/geronb/gbr019

Edkins, G. \& Pollock, C. (1997), 'The Influence of Sustained Attention on Railway Accidents', Accident Analysis and Prevention, 29: 533-9. https://doi.org/10.1016/S0001-4575(97)00033-X

Feeney, J., Kamiya, Y., Robertson, I. H. \& Kenny, R. A. (2013), 'Cognitive Function is Preserved in Older Adults with a Reported History of Childhood Sexual Abuse', Journal of Traumatic Stress, 26: 735-43. https://doi.org/10.1002/jts.21861

Fineberg, N. A., Haddad, P. M., Carpenter, L., Gannon, B., Sharpe, R., Young, A. H., Joyce, E., Rowe, J., Wellsted, D. \& Nutt, D. J. (2013), 'The Size, Burden and Cost of Disorders of the Brain in the UK', Journal of Psychopharmacology, 27: 761-70. https://doi.org/10.1177/0269881113495118

Greene, C., Bellgrove, M. A., Gill, M. \& Robertson, I. H. (2009), 'Noradrenergic Genotype Predicts Lapses in Sustained Attention', Neuropsychologia, 47: 591-4. https://doi.org/10.1016/j.neuropsychologia.2008.10.003

Grefkes, C., Wang, L. E., Eickhoff, S. B. \& Fink, G. R. (2010), 'Noradrenergic Modulation of Cortical Networks Engaged in Visuomotor Processing', Cerebral Cortex, 20: 783-97. https://doi.org/10.1093/cercor/bhp144

Kessler, R. C., Chiu, W. T., Demler, O. \& Walters, E. E. (2005), 'Prevalence, Severity, and Comorbidity of 12-Month DSM-IV Disorders in the National Comorbidity Survey Replication', Archives of General Psychiatry, 62: 617-27. https://doi.org/10.1001/archpsyc.62.6.617

Manly, T., Robertson, I. H., Galloway, M. \& Hawkins, K. (1999), 'The Absent Mind: Further Investigations of Sustained Attention to Response', Neuropsychologia, 37: 661-70. https://doi.org/10.1016/S0028-3932(98)00127-4

Manly, T., Anderson, V., Nimmo-Smith, I., Turner, A., Watson, P. \& Robertson, I. H. (2001), 'The Differential Assessment of Children's Attention: The Test of Everyday Attention for Children (TEA-Ch), Normative Sample and ADHD Performance', Journal of Child Psychology and Psychiatry, 42: 1-10. https://doi.org/10.1111/1469-7610.00806 
Manly, T., Hawkins, K., Evans, J., Woldt, K. \& Robertson, I. H. (2002), 'Rehabilitation of Executive Function: Facilitation of Effective Goal Management on Complex Tasks Using Periodic Auditory Alerts', Neuropsychologia, 40: 271-81. https://doi.org/10.1016/S0028-3932(01)00094-X

Manly, T., Owen, A. M., Datta, A., Lewis, G., Scott, S., Rorden, C., Pickard, J. \& Robertson, I. H. (2003), 'Enhancing the Sensitivity of a Sustained Attention Task to Frontal Damage. Convergent Clinical and Functional Imaging Evidence', Neurocase, 9:340-9. https://doi.org/10.1076/neur.9.4.340.15553

Mattarella-Micke, A., Mateo, J., Kozak, M. N., Foster, K. \& Beilock, S. L. (2011), 'Choke or Thrive? The Relation Between Salivary Cortisol and Math Performance Depends on Individual Differences in Working Memory and Math-anxiety', Emotion, 11: 1000-5. https://doi.org/10.1037/a0023224

Molenberghs, P., Gillebert, C. R., Schoofs, H., Dupont, P., Peeters, R. \& Vandenberghe, R. (2009), 'Lesion Neuroanatomy of the Sustained Attention to Response Task', Neuropsychologia, 47: 2866-75. https://doi.org/10.1016/j.neuropsychologia.2009.06.012

Murphy, P. R., Robertson, I. H., Balsters, J. H. \& O’Connell, R. G. (2011), 'Pupillometry and P3 Index the Locus Coeruleus-Noradrenergic Arousal Function in Humans', Psychophysiology, 48: 1532-43. https://doi.org/10.1111/j.1469-8986.2011.01226.x

Murphy, P. R., O’Connell, R. G., O’Sullivan, M., Robertson, I. H. \& Balsters, J. H. (2014), 'Pupil Diameter Covaries with BOLD Activity in Human Locus Coeruleus', Human Brain Mapping, 35: 4140-54. https://doi.org/10.1002/hbm.22466

O’Connell, R. G., Bellgrove, M. A., Dockree, P. M., Lau, A., Fitzgerald, M. \& Robertson, I. H. (2008), 'Self-Alert Training: Volitional Modulation of Autonomic Arousal Improves Sustained Attention', Neuropsychologia, 46: 1379-90. https://doi.org/10.1016/j.neuropsychologia.2007.12.018

O’Connor, C., Robertson, I. H. \& Levine, B. (2011), 'The Prosthetics of Vigilant Attention: Random Cuing Cuts Processing Demands', Neuropsychology, 25: 535-43. https://doi.org/10.1037/a0022767

Robertson, I. H. (2013), 'A Noradrenergic Theory of Cognitive Reserve: Implications for Alzheimer's Disease', Neurobiology of Aging, 34: 298-308.

https://doi.org/10.1016/j.neurobiolaging.2012.05.019

Robertson, I. (2016) The Stress Test: How Pressure Can Make You Stronger and Sharper (London, Bloomsbury).

Robertson, I. H., Ward, T., Ridgeway, V. \& Nimmo-Smith, I. (1996), 'The Structure of Normal Human Attention: The Test of Everyday Attention', Journal of the International Neuropsychological Society, 2: 525-34. https://doi.org/10.1017/S1355617700001697

Robertson, I. H., Manly, T., Andrade, J., Baddeley, B. T. \& Yiend, J. (1997), “ “Oops!”: Performance Correlates of Everyday Attentional Failures in Traumatic Brain Injured and Normal Subjects', Neuropsychologia, 35: 747-58. https://doi.org/10.1016/S0028-3932(97)00015-8

Salomone, S., Fleming, G. R., Shanahan, J. M., Castorina, M., Bramham, J., O'Connell, R. G. \& Robertson, I. (2015), 'The Effects of a Self-Alert Training (SAT) Program in Adults with ADHD', Frontiers in Human Neuroscience, 9. https://doi.org/10.3389/fnhum.2015.00045

Sara, S. J. (2009), 'The Locus Coeruleus and Noradrenergic Modulation of Cognition', Nature Reviews Neuroscience, 10: 211-23. https://doi.org/10.1038/nrn2573

Seery, M. D., Holman, E. A. \& Silver, R. C. (2010a), 'Whatever Does Not Kill Us: Cumulative Lifetime Adversity, Vulnerability, and Resilience', Journal of Personality and Social Psychology, 99, 1025-41. https://doi.org/10.1037/a0021344

Seery, M. D., Leo, R. J., Holman, E. A. \& Cohen Silver, R. (2010b), 'Lifetime Exposure to Adversity Predicts Functional Impairment and Healthcare Utilization Among Individuals with Chronic Back Pain', Pain, 150: 507-15. https://doi.org/10.1016/j.pain.2010.06.007

Singh-Curry, V. \& Husain, M. (2009), 'The Functional Role of the Inferior Parietal Lobe in the Dorsal and Ventral Stream Dichotomy', Neuropsychologia, 47: 1434- 48.

https://doi.org/10.1016/j.neuropsychologia.2008.11.033 
Smilek, D., Carriere, J. S. A. \& Cheyne, J. A. (2010), 'Failures of Sustained Attention in Life, Lab, and Brain: Ecological Validity of the SART', Neuropsychologia, 48: 2564-70. https://doi.org/10.1016/j.neuropsychologia.2010.05.002

Stern, R. A., Silva, S. G., Chaisson, N. \& Evans, D. L. (1996), 'Influence of Cognitive Reserve on Neuropsychological Functioning in Asymptomatic Human Immunodeficiency Virus-1 Infection', Archives of Neurology, 53: 148-53. https://doi.org/10.1001/archneur.1996.00550020052015

The Highways Agency (2016) Design Manual for Roads and Bridges. Road Geometry (London, HM Stationery Office).

Twenge, J. M., Gentile, B., Dewall, C. N., Ma, D., Lacefield, K. \& Schurtz, D. R. (2010), 'Birth Cohort Increases in Psychopathology Among Young Americans, 1938-2007: A Cross-temporal Meta-analysis of the MMPI', Clinical Psychology Review, 30: 145-54. https://doi.org/10.1016/j.cpr.2009.10.005

Wilson, R. S., Nag, S., Boyle, P. A., Hizel, L. P., Yu, L., Buchman, A. S., Schneider, J. A. \& Bennett, D. A. (2013), 'Neural Reserve, Neuronal Density in the Locus Ceruleus, and Cognitive Decline', Neurology, 80: 1202-8. https://doi.org/10.1212/WNL.0b013e3182897103

Yerkes, R. M. \& Dodson, J. D. (1908), 'The Relation of Strength of Stimulus to Rapidity of Habitformation' Journal of Comparative and Neurological Psychology, 18: 459-82. https://doi.org/10.1002/cne.920180503

Note on the author: Ian Robertson (www.ianrobertson.org) is Emeritus Professor of Psychology at Trinity College Dublin, Visiting Professor at University College Dublin and at the University of Texas at Dallas (Center for BrainHealth). His book The Stress Test: How Pressure Can Make You Stronger and Sharper, published by Bloomsbury, is an account for the lay reader of 35 years of research which are summarised in the book and more briefly in this paper. iroberts@tcd.ie

To cite the article: Ian H. Robertson (2017), 'The stress test: Can stress ever be beneficial?', Journal of the British Academy, 5: 163-176.

DOI https://doi.org/10.85871/jba/005.163

This article is licensed under a

Creative Commons Attribution-NonCommercial-NoDerivs 3.0 Unported License.

Journal of the British Academy (ISSN 2052-7217) is published by

The British Academy - the national academy for the humanities and social sciences.

10-11 Carlton House Terrace, London, SW1Y 5AH

www.britishacademy.ac.uk 